\title{
Electronic Band Structure of Gd: A Consistent Description
}

\author{
K. Maiti, ${ }^{1}$ M. C. Malagoli, ${ }^{1}$ E. Magnano, ${ }^{2}$ A. Dallmeyer, ${ }^{1}$ and C. Carbone ${ }^{1}$ \\ ${ }^{1}$ Institut für Festkörperforschung, Forschungszentrum Jülich, D-52425 Jülich, Germany \\ ${ }^{2}$ Laboratorio Nazionale TASC, Istituto Nazionale per la Fisica della Materia, Strada Statale 14-Km.163.5, \\ Basovizza, I-34012 Trieste, Italy
}

(Received 2 August 2000)

\begin{abstract}
The dispersion of the Gd $(5 d 6 s)$-valence bands has been investigated by means of spin- and angleresolved photoemission. The spin analysis of various spectral features shows that their weak dispersion and unusual broadening is due to the photoelectron lifetime rather than to correlation induced band narrowing as previously proposed. These results resolve a long-standing discrepancy between theoretical and experimental descriptions of the rare earth band structure.
\end{abstract}

DOI: $10.1103 /$ PhysRevLett.86.2846

The unique electronic and magnetic properties of lanthanides result from the coexistence of both highly localized and delocalized levels in the valence region. While the $4 f$ electrons maintain an atomic character in these metals, the $(5 d 6 s)$ states form the metallic bonding and mediate the indirect exchange interaction among the $4 f$ moments.

Photoemission spectroscopy gives direct access to the excited- and ground-state configurations of the $4 f$ shell that are indeed well understood for the lanthanide metals [1]. On the contrary, no satisfactory picture of the $(5 d 6 s)$ states exists so far. Even fundamental questions such as the accuracy of the band structure methods for the ground-state description or the influence of many-body effects on the excitation spectra have not yet found an answer. In fact, band structure calculations based on the local spin density approximations (LSDA) predict a large bandwidth for the $(5 d 6 s)$ states in rare earths similar to that of the preceding elements (alkali and alkaline earth metals) in the Periodic Table. These latter cases [2-5] exhibit strongly dispersive photoemission features that are reasonably well interpreted within the paradigms based on effective single-particle approximations, although a quantitative description requires detailed consideration of many-body effects. In sharp contrast, angle-resolved photoemission from lanthanides does not display the expected dispersive behavior of the $(5 d 6 s)$-bulk states. Various observations [6-8] seem to indicate a failure of the effective singleparticle descriptions to the $(5 d 6 s)$ photoemission and suggest the importance of correlation effects.

The situation is well illustrated by the case of Gd, which is the most extensively studied lanthanide element for its importance as a prototypical Heisenberg ferromagnet $\left(T_{C}=293 \mathrm{~K}\right)$. Photoemission studies report the energy separations between the $\Gamma_{4^{-}}-\Gamma_{1^{+}}$points [9] and $\Gamma_{4^{-}}-$ $A_{1}$ points [8] to be by about a factor of 2 smaller than that predicted by the state-of-the-art calculations $[10,11]$ [shown in Fig. 1(a) for the ferromagnetic ground state [12] ]. Correspondingly, only a significantly weak energy dispersion of the $\Delta_{2}$ band is observed by varying the photon energy $[8,9]$. While this latter observation, separately taken, could possibly be explained by momentum broad-
PACS numbers: 71.20.Eh, 71.27. $+\mathrm{a}, 75.25 .+\mathrm{z}, 79.60 . \mathrm{Bm}$

ening [2], the concomitant discrepancy between the experimental and theoretical bandwidth leads to attribute [8] both the effects to strong electron-electron interactions. Notably, such band narrowing in Gd appears to be much larger than that in the strongly correlated $3 d$ transition metals [13-15], an unusual fact that has not been understood so far.

In order to clarify the inconsistency between the experimental and theoretical descriptions in rare earths, we investigate the electronic band structure of $\mathrm{Gd}$ by means of spin- and angle-resolved photoemission spectroscopy. Spin-resolved spectra reveal an evolution of the spectral line shape as a function of photon energy that cannot be visualized in the spin-integrated measurements. Consideration of the photoelectron lifetime convoluted to the LSDA band structure results account well for the observed spectral evolution. The present results thus resolve the long-standing discrepancy between photoemission and band theory of Gd and are supposedly of direct relevance for the other rare earth metals and related $(\mathrm{Sc}, \mathrm{Y}, \mathrm{La}$, etc.) elements as well.

The experiments were carried out on the TGM5 wigglerundulator beam line at BESSY I, Berlin. The overall experimental resolution was $160 \mathrm{meV}$ with angular resolution better than $\pm 0.5^{\circ}$. Gd(0001) films of 30 monolayer (ML) thickness were epitaxially grown on a clean W(110) surface at room temperature and subsequently annealed at $600 \mathrm{~K}$. The crystalline structure of the films was confirmed by very sharp LEED patterns.

The spin-integrated normal emission spectra of the Gd valence band at room temperature (paramagnetic state) are shown in Fig. 1(b). The nondispersive sharp feature close to the Fermi level $\left(E_{F}\right)$ is due to emission from $5 d_{z^{2}}$ surface states. The broader signal around $1.8 \mathrm{eV}$ is the signature of the $(5 d 6 s)$-bulk band. This feature exhibits the binding energy extrema corresponding to the $\Gamma$ point of the Brillouin zone at photon energies of about 34 and $67.5 \mathrm{eV}$. This is consistent with the expectation from a free-electron-like model for the final states, with an inner potential $V_{0}$ assumed to be $-8 \mathrm{eV}$. Thus, the other extreme, $A$, should be reached at about $50 \mathrm{eV}$ photon 

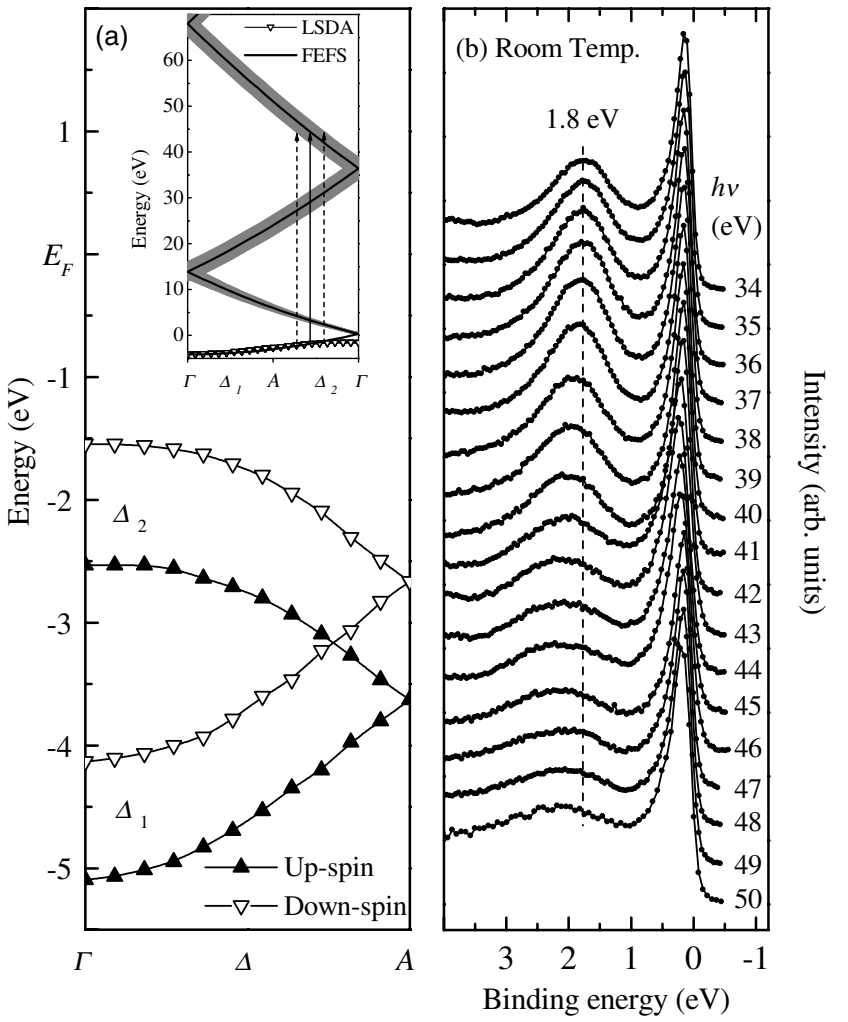

FIG. 1. (a) Band dispersion in the ferromagnetic ground state of Gd along $\Gamma A$ [12]. The up-spin and down-spin bands are represented by solid up triangles and open down triangles, respectively. The inset shows the calculated ground state and the free-electron final state band (FEFS) along $\Gamma A$ used in the simulation. The shaded region schematically represents the energy uncertainty in the final state bands due to the photoelectron lifetime effects. (b) Spin-integrated normal emission valence band spectra of $30 \mathrm{ML} \mathrm{Gd(0001)}$ at room temperature. The dashed line represents the energy position of the feature at $\Gamma$.

energy. Consistent with previous work [8], the spectra in Fig. 1(b) exhibit a small energy shift of the $\Delta_{2}$ emission peak for photon energy increasing from 34 to $50 \mathrm{eV}$, which is less than half of the energy dispersion expected for the magnetic ground state [see Fig. 1(a)]. It should also be noted that the linewidth of the $\Delta_{2}$ spectral feature increases substantially for photon energies beyond $40 \mathrm{eV}$.

In the spectra of the ferromagnetic state at $120 \mathrm{~K}$ [see Fig. 2(a)], the $\Delta_{2}$ band emission at $34 \mathrm{eV}$ photon energy exhibits a pair of exchange-split features at about 2.3 and $1.4 \mathrm{eV}$. Their separation corresponds to an exchange splitting of about $0.9 \mathrm{eV}$ in close agreement with the theoretical results [11]. At higher photon energies, the intensity of the sharp down-spin feature appears to gradually shift as is most evident in the spectra between 40 and $44 \mathrm{eV}$. Interestingly, the spectra at photon energies above $44 \mathrm{eV}$ are, however, again quite similar to those at $34 \mathrm{eV}$. In previous photoemission studies [8], the bandwidth of the up-spin and down-spin $\Delta_{2}$ bands was estimated to be about $0.5 \mathrm{eV}$ from the spectra virtually identical to the ones presented here. We show in the following that the knowledge of the

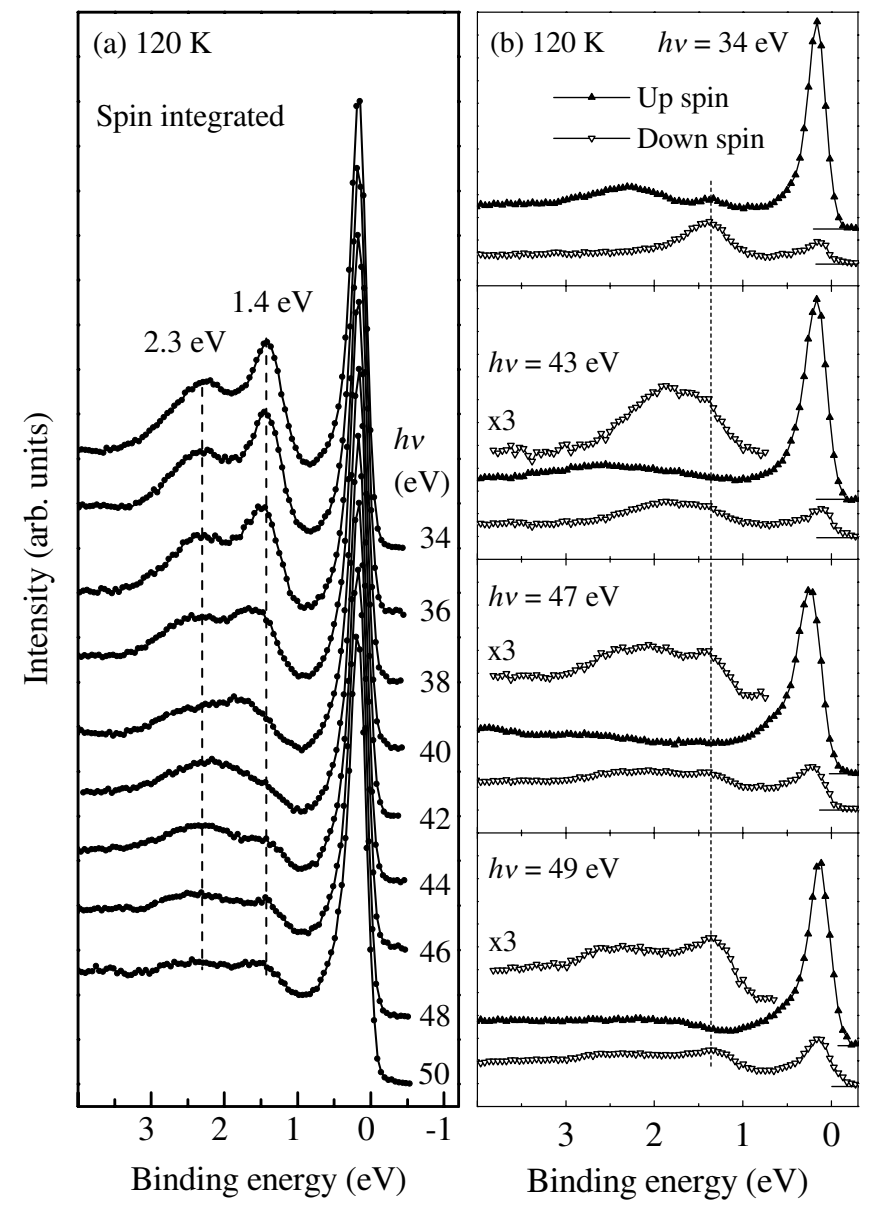

FIG. 2. (a) Spin-integrated and (b) spin-resolved normal emission valence band spectra of $30 \mathrm{ML} \mathrm{Gd(0001)} \mathrm{film} \mathrm{at} 120 \mathrm{~K}$ as a function of photon energy. The dashed line represents the energy position of the spectral feature at $\Gamma$.

spin characters of the various features leads to very different conclusions.

Spin-resolved spectra at some selected photon energies are shown in Fig. 2(b). The surface feature is predominantly of up-spin character with small down-spin contributions, as already known from previous studies [16]. The $\Delta_{2}$ emission at $34 \mathrm{eV}$ photon energy is resolved into an upand down-spin component at about 2.3 and $1.4 \mathrm{eV}$, respectively. The up-spin component is broader than the down-spin emissions and slowly loses its identity at higher photon energies. This may be attributed primarily to the spin and energy dependence of the lifetime broadening of the photoholes. The down-spin component exhibits distinct changes with the photon energy on which we will concentrate the following discussion.

For the sake of clarity we expand the down-spin component in the figure. It is clear that the increase in photon energy leads to an overall spectral weight transfer to higher binding energies. The line shape can roughly be described as resulting from a strongly dispersive feature along with a nearly nondispersive component at about $1.4 \mathrm{eV}$. These spectral modifications of the down-spin emission could not 
be identified in the spin-integrated measurements [as in Fig. 2(a)] due to the unresolved and overlapping emissions from both the spin channels. Most significantly, for photon energies sampling the $k$ points closer to $A$ (i.e., $45-50 \mathrm{eV}$ ), the peak around $2.5 \mathrm{eV}$ binding energy turns out to have predominantly down-spin character. In the spin-integrated study, this structure was assumed to be due to up-spin states [8] and led thus to an incorrect estimate of the band dispersion. While the spectral shift of the high binding energy component of the spectra resembles well the dispersion of the LSDA bands, the presence of a nondispersive feature does not have any correspondence in the theoretical results. The degeneracy with the bulk bands and its insensitivity to the surface contaminations rule out the possibility of surface character of this feature.

It is notable here that photoemission is intrinsically a many-body process. The shape, intensity, and energy position of the spectral features are influenced by various dynamical interactions in the solid that are not included in effective single-particle models. The corrections due to the many-body interactions are often referred to as self-energy corrections to the retarded Green's function, $G(k, E)=1 /\left\{E-\epsilon_{k}-\Sigma(k, E)\right\}$ where $\epsilon_{k}$ is the energy eigenvalue from the effective single-particle calculations and the complex function $\Sigma(k, E)$ is the self-energy. $\Sigma(k, E)$ contains the contributions from the electron correlations. The real and imaginary part of $\Sigma(k, E)$ determine the position and width of the spectral function (imaginary part of the Green's function), respectively. In addition, the spectral width will be significantly influenced by the lifetime of the photoholes and the photoelectrons. The increase in available decay channels for the holes at higher binding energies introduces an energy-dependent lifetime broadening. The decay of the photoelectron wave functions due to various inelastic scattering processes while traveling through the solid introduces a momentum broadening as indicated in the inset of Fig. 1(a) [2]. Thus, the spectral function will be a convolution of the many-body spectral function $S^{\prime}\left(k_{\perp}^{\prime}, \epsilon_{h}\right)$ with the hole and electron lifetime broadening functions and can be expressed as $S\left(k_{\perp}, E\right) \propto \iint d \epsilon_{h} d k_{\perp}^{\prime} S^{\prime}\left(k_{\perp}^{\prime}, \epsilon_{h}\right)\left[\Gamma_{h} /\left\{\left(\epsilon_{h}-\right.\right.\right.$ $\left.\left.E)^{2}+\Gamma_{h}^{2}\right\}\right]\left[\Gamma_{k} /\left\{\left(k_{\perp}^{\prime}-k_{\perp}\right)^{2}+\Gamma_{k}^{2}\right\}\right]$ where $\Gamma_{k}$ is the half width at half maximum (HWHM) of the Lorentzian representing the momentum broadening which is the inverse of the electron escape depth, $\lambda\left(\Gamma_{k}=\frac{1}{2 \lambda}\right) . \Gamma_{h}$ is HWHM of the Lorentzian for the holes and often being found [17] to be of the form, $\Gamma_{h}=\Gamma_{0}+\alpha \times \mathrm{BE}^{2}$; $\mathrm{BE}=$ binding energy, suggesting a Fermi liquid behavior. $k_{\perp}$ is the perpendicular component of the reciprocal lattice vector; the parallel component $k_{\|}$is zero for normal emission geometry.

In order to investigate the influence of the momentum broadening, we simulate the down-spin spectral functions using the calculated ground-state band and the free-electron-like final states as shown in the inset of Fig. 1(a). The symmetry conservation rules for the hcp lattice [18] are considered in the calculation. Thus, we sample the $\Delta_{1}$ and $\Delta_{2}$ bands shown in the inset of Fig. 1(a) for the calculation. The values of $\Gamma_{0}$ and $\alpha$ are fixed to be $0.15 \mathrm{eV}$ and $0.075 \mathrm{eV}^{-1}$; these values are similar to those observed in this [17] and other systems [19]. The experimental resolution is accounted for via a Gaussian convolution of the calculated spectral functions. Thus, the only parameter $\Gamma_{k}$ is varied to simulate the experimental spectra at all the photon energies. It is necessary to shift the calculated dispersions by about $0.2 \mathrm{eV}$ towards $E_{F}$ to compare with the experimental spectra. The calculated results for $\Gamma_{k}=0.17 \pm 0.03 \AA^{-1}$ are shown in Fig. 3(a) by solid lines along with the experimental down-spin spectra at different photon energies. While there is a little discrepancy in the relative intensity of the features, the spectral weight transfer and the presence of a nondispersive feature is clearly manifested in the simulated spectra consistently with the experimental ones. From the band structure calculations [11], it is known that the character of the bands close to $E_{F}(\mathrm{BE} \leq 1.5 \mathrm{eV})$ is dominated by $5 d$ states and the states at higher binding energies are essentially of $6 s$ character. Since the photoemission cross section of the $5 d$ states are larger than the $6 s$ states at these photon energies [20], the larger intensity of the $1.4 \mathrm{eV}$ feature in the experiment may be attributed to the neglect of matrix element effects in our calculations.

Thus, considering the limitations and the simplicity of the model, the agreement with the experimental spectra

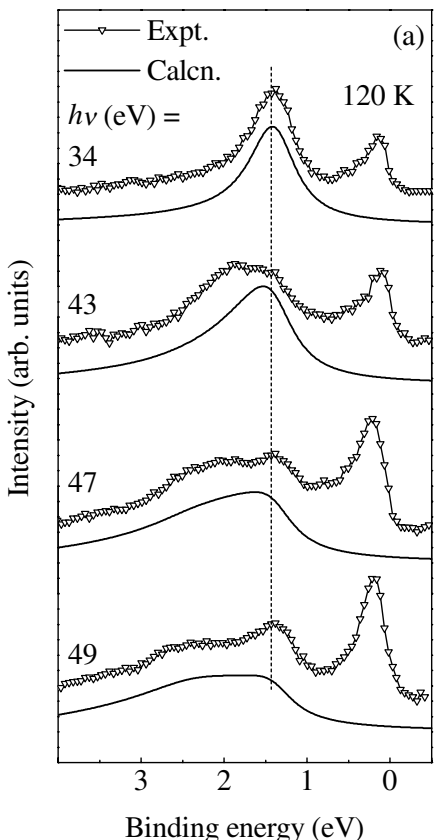

Binding energy $(\mathrm{eV})$

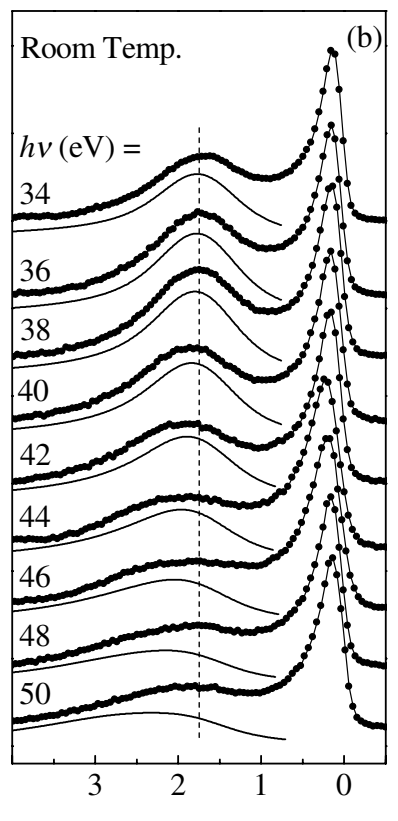

Binding energy $(\mathrm{eV})$
FIG. 3. Experimental (a) low temperature down-spin spectra and (b) room temperature spin-integrated spectra at different photon energies represented by the open down triangles and solid circles, respectively. The solid lines show the corresponding simulated spectral functions from the LSDA results. The dashed line represents the energy position of the spectral feature at $\Gamma$. 
is remarkable. The value of $\Gamma_{k}$ corresponds to the escape depth, $\lambda \sim 3 \AA$ which is a reasonable estimate [21] at these photon energies. Therefore, it appears that the weak dispersion of the $\Delta_{2}$ band is essentially due to the photoelectron lifetime effect rather than the strong electron correlation as previously proposed. The contribution from each $k$ point close to $\Gamma$ is about $10 \%$ of the one from $k$ points close to $A$, when the photon energy corresponds to the $A$ point. Considering relatively flat bands between $\Gamma$ and $1 / 2 \times(\Gamma-A)$, it can be easily realized that they give rise to a sizable spectral contribution (i.e., a nondispersive feature) at all photon energies. The same analysis could also be carried out for the up-spin spectra. However, the large spin and energy-dependent lifetime broadening makes it difficult to visualize the changes. We have calculated all the spectra at room temperature with the same parameters as shown in Fig. 3(b) exhibiting a remarkable agreement with the experimental spectra. Interestingly, the unusual spectral broadening above $40 \mathrm{eV}$ could be simulated exactly. The band dispersion at room and low temperatures as a function of $k_{\|}$could also be simulated consistently with the experimental observations with the same parameters. Such consistency with the spectra probed at different experimental conditions provides strong confidence to the estimated parameters.

The small shift of the calculated spectra with respect to the experimental ones might be related to the many-body effects, similar to alkali [3] and alkaline earths [5] as well as transition metals [13-15]. It has been shown via LSDA $+U$ calculations [22] that the electron correlation is important in determining the exchange splitting of the highly localized $4 f$ states which cannot be accounted by the LSDA methods. However, the electron correlation within the $5 d$ electrons plays only a minor role in the dispersion of the valence states [23].

In summary, this study resolves the long-standing conflict between theory and experiment on the electronic band structure of rare earth metal systems. Spin-resolved measurements establish a clear correspondence between the experimental band dispersion as it is probed by angleresolved photoemission and band structure calculations. The results point out the importance of photoelectron lifetime in determining the experimental band dispersions and significantly reassess the role of correlation effects on the $(5 d 6 s)$ states in rare earths.

The authors thank Professor S. Blügel, as well as Professor A. I. Lichtenstein and P. Kurz for useful discus- sions. K. M. acknowledges the Alexander von Humboldt Foundation, the Federal Republic of Germany, for financial assistance.

[1] J. K. Lang and Y. Baer, J. Magn. Magn. Mater, 15-18, 1227 (1980); J. K. Lang, Y. Baer, and P. A. Cox, J. Phys. F 11, 121 (1981); P. A. Cox, J. K. Lang, and Y. Baer, J. Phys. F 11, 113 (1981).

[2] E. Jensen and E. W. Plummer, Phys. Rev. Lett. 55, 1912 (1985); I.-W. Lyo and E. W. Plummer, Phys. Rev. Lett. 60, 1558 (1988).

[3] K. W.-K. Shung and G. D. Mahan, Phys. Rev. Lett. 57, 1076 (1986); K. W.-K. Shung, B. E. Sernelius, and G. D. Mahan, Phys. Rev. B 36, 4499 (1987).

[4] B. S. Itchkawitz, I.-W. Lyo, and E. W. Plummer, Phys. Rev. B 41, 8075 (1990).

[5] E. Jensen et al., Phys. Rev. B 30, 5500 (1984); R. A. Bartynski et al., Phys. Rev. B 33, 3644 (1986).

[6] E. Weschke et al., Phys. Rev. B 58, 3682 (1998).

[7] S. C. Wu et al., Phys. Rev. B 44, 13720 (1991).

[8] B. Kim et al., Phys. Rev. Lett. 68, 1931 (1992).

[9] F. J. Himpsel and B. Reihl, Phys. Rev. B 28, 574 (1983).

[10] B. C. H. Krutzen and F. Springelkamp, J. Phys. Condens. Matter 1, 8369 (1989); W. M. Temmerman and P. A. Sterne, J. Phys. Condens. Matter 2, 5529 (1990); M. Heinemann and W. M. Temmerman, Phys. Rev. B 49, 4348 (1994); O. Eriksson et al., Phys. Rev. B 52, 4420 (1995).

[11] J. Sticht and J. Kübler, Solid State Commun. 53, 529 (1985); R. Wu, C. Li, A. J. Freeman, and C.L. Fu, Phys. Rev. B 44, 9400 (1991).

[12] P. Kurz, Ph.D. thesis, Köln, 2000. The bandwidth in this calculation is similar to those published earlier [10,11].

[13] A. Santoni and F. J. Himpsel, Phys. Rev. B 43, 1305 (1991).

[14] W. Clemens et al., Solid State Commun. 81, 739 (1992).

[15] W. Eberhardt and E. W. Plummer, Phys. Rev. B 21, 3245 (1980); F. J. Himpsel, J. A. Knapp, and D. E. Eastman, Phys. Rev. B 19, 2919 (1979).

[16] Dongqi Li et al., Phys. Rev. B 51, 13895 (1995).

[17] K. Maiti, M.C. Malagoli, A. Dallmeyer, and C. Carbone (unpublished).

[18] J. F. Cornwell, Phys. Kondens. Mater. 4, 327 (1966); F. J. Himpsel and D. E. Eastman, Phys. Rev. B 21, 3207 (1980).

[19] T. Valla et al., Phys. Rev. Lett. 83, 2085 (1999).

[20] J. J. Yeh and I. Lindau, At. Data Nucl. Data Tables 32, 1 (1985).

[21] R. Kammerer et al., Solid State Commun. 41, 435 (1982).

[22] A. B. Shick, A. I. Liechtenstein, and W.E. Pickett, Phys. Rev. B 60, 10763 (1999).

[23] A.I. Lichtenstein (private communication). 